\title{
Some Applications of Holography to Study Strongly Correlated Systems
}

\author{
Neha Bhatnagar \\ Department of Physics, Banaras Hindu University, \\ Varanasi-221005, India, \\ bhtngr.neha@gmail.com,
}

\begin{abstract}
In this work, we study the transport coefficients of strongly coupled condensed matter systems using gauge/gravity duality (holography). We consider examples from the real world and evaluate the conductivities from their gravity duals. Adopting the bottom-up approach of holography, we obtain the frequency response of the conductivity for $(1+1)$-dimensional systems. We also evaluate the DC conductivities for non-relativistic condensed matter systems with hyperscaling violating geometry.
\end{abstract}

Keywords: Holography, bottom-up approach, strongly coupled systems

\section{Introduction}

Recently, the discovery of high $T_{c}$ superconductors and strange metals challenged the old concept of Fermi-liquid theory and the BCS (Bardeen Cooper Schrieffer) theory. These newly found materials are considered inherently strongly coupled with no well-defined quasi-particles. Thus, perturbative methods and conventional approaches fail to probe them completely. The remarkable progress in this area occurs with the advent of AdS/CFT duality[1]. The duality relates d-dimensional strongly coupled gauge theory with weakly coupled gravity theory in $(d+1)$-dimensions. The extra dimension in bulk corresponds to the energy scale of the boundary theory. Holography relates high $T_{c}$ superconductivity with the spontaneously broken U(1) gauge symmetry modeled by introducing complex scalar field in the gravity background $[2,3]$. The next significant development in this area occurs with the introduction of momentum dissipation term in the gravity action and obtain finite DC conductivity. In this work, we discuss the transport coefficients for two different strongly correlated systems using the holographic approach in a simplified manner.

\section{2 (1+1)-dimensional systems}

We first explore (1+1)-dimensional systems using holography. Lower dimensional systems such as nanowires, Luttinger liquids, and spin chains are useful for technology development but are difficult to analyse theoretically because of strong correlated nature. Applying the holographic approach, we capture the frequency response of the conductivity for the system. The gravity action in the soft-wall model is given by,

$$
S=\int d^{3} x \sqrt{-g} e^{-2 \phi}\left(\frac{1}{2 \kappa^{2}}(R-2 \Lambda)+\frac{1}{4 g^{2}} F^{2}\right)
$$


where, $F^{2}$ is the gauge field strength, $\kappa^{2}=8 \pi G_{3}=1$ and $\Lambda=-2 / L^{2}$. Here the dilaton field $\phi$, softly truncates the IR boundary. The equations of motion are given in the form,

$$
R_{\mu \nu}-\frac{1}{2} g_{\mu \nu} R-\Lambda g_{\mu \nu}=T_{\mu \nu}, \quad \text { and } \quad \nabla_{\mu}\left(e^{-2 \phi} F^{\mu \nu}\right)=0
$$

where $T_{\mu \nu}$ is the energy-momentum tensor. We consider the metric ansatz as $[4,5]$,

$$
d s^{2}=-f(r) d t^{2}+r^{2} d x^{2}+\frac{d r^{2}}{f(r)}
$$

$$
\text { where } f(r)=r^{2}-r_{h}^{2}+\frac{\mu^{2}}{2 r^{2}} \ln \left(\frac{r_{h}}{r}\right) \text {, and } \quad A_{t}^{\prime}=\mu e^{2 \phi} \ln \left(\frac{r_{h}}{r}\right) \text {. }
$$

In this model, $\mu$ corresponds to the chemical potential and we set, $r_{h}=1$ (radius of black hole horizon). We introduce perturbations in the gauge field and metric tensors in the Fourier space as, $A_{x}(r, t)=a_{x}(r) e^{-i \omega t}$ and $\mathbf{h}_{x t}(r, t)=h_{x t}(r) e^{-i \omega t}$. The linearised equations of motion are given by,

$$
\begin{aligned}
h_{t}^{\prime x}-A_{t}^{\prime} z^{2} a_{x} & =0, \\
a_{x}^{\prime \prime}+\left(\frac{f^{\prime}}{f}+\frac{1}{r}-2 \phi^{\prime}\right) a_{x}^{\prime}-\frac{A_{t}^{\prime} h_{t}^{\prime x}}{f}+\frac{\omega^{2}}{f^{2}} a_{x} & =0 .
\end{aligned}
$$

The boundary asymptotics of the gauge field takes the form, $a_{x}=-a_{x}^{(0)} \ln r+a_{x}^{(1)}$. Now applying the in-going boundary condition to maintain the casual behavior the optical conductivity is obtained using $[4,5], \sigma(\omega)=\frac{i a_{x}^{(1)}}{\omega a_{x}^{(0)}}=\left.\frac{i\left(a_{x}+r a_{x}^{\prime} r \ln [r]\right)}{\omega r a_{x}^{\prime}}\right|_{r \rightarrow \epsilon}$. We consider two different dilaton profiles, $\phi(r)=\frac{1}{r}$ (Model-I) and $\phi(r)=\frac{1}{r^{2}}$ (Model-II) to investigate the frequency response of the system with varying chemical potential shown in Fig. 1. and Fig. 2 respectively.
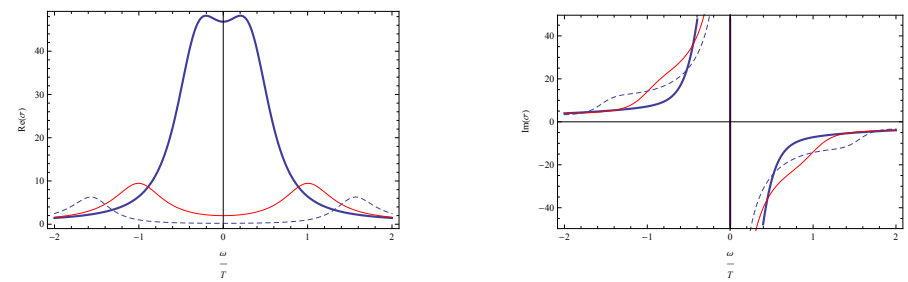

Fig. 1: Frequency response of the conductivity for $\phi=\frac{1}{r}$ (Model-I)

We attempt to capture the response of $(1+1)$-dimensional boundary theory with finite chemical potential. At lower frequency, we obtained Drude peak indicating the normal phase of the holographic system. With the increase in $\mu$, we observe flattening of the peak in $R e[\sigma]$. Different dilaton profiles correspond to different condensate in the holographic superconductor[6]. Applying the soft-wall model for our work gave us the freedom to explore the specific features of strongly coupled condensed matter systems in a phenomenological simple way. We have captured the Fermi-Luttinger liquid behavior of the boundary theory using the soft-wall model for two different dilaton profiles. 

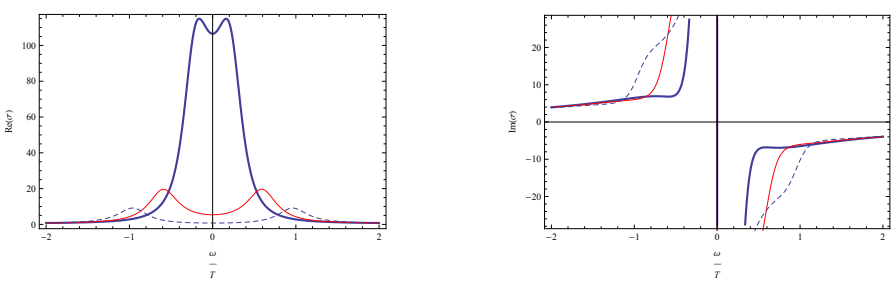

Fig. 2: Frequency response of the conductivity for $\phi=\frac{1}{r^{2}}$ (Model-II)

\section{Non-relativistic condensed matter systems with hyperscaling violation}

We investigate the DC conductivities for non-relativistic condensed matter system using (3+1)-dimensional EMD (Einstein-Maxwell-Dilaton) theory with hyperscaling violation [7]. The gravity action is given by,

$$
S=\int d^{4} x \sqrt{-g}\left(R-\frac{1}{2}(\partial \phi)^{2}+V(\phi)-\frac{1}{4} \sum_{i=1}^{2} e^{\lambda_{i} \phi} F_{i}^{2}-e^{\lambda_{3} \phi} \sum_{i=1}^{2} \partial \chi_{i}^{2}\right)
$$

where, $F_{i}$ as two different gauge fields (one for introducing Liftshitz symmetry and the other to incorporate charge in the system), $V(\phi)=-2 \Lambda e^{\lambda_{0} \phi}$ (Liouville-like dilaton potential) and axionic fields (for momentum dissipation) are given as, $\chi_{1}=\alpha x$ and $\chi_{2}=\alpha y$. We obtain the following equations of motion for our holographic set-up,

$$
\begin{gathered}
R_{\mu \nu}=\frac{1}{2} \partial_{\mu} \phi \partial_{\nu} \phi+\frac{1}{2} e^{\lambda_{3} \phi}\left(\partial_{\mu} \chi_{i} \partial_{\nu} \chi_{i}\right)+\Lambda e^{\lambda_{0} \phi} g_{\mu \nu}+\sum_{i=1}^{2} \frac{1}{2} e^{\lambda_{i} \phi}\left(F_{i \mu \rho} F_{i \nu}^{\rho}-\frac{1}{4} F_{i}^{2} g_{\mu \nu}\right) \\
\square \phi=\frac{1}{2} \lambda_{3} \sum_{i=1}^{2}\left(\partial \chi_{i}\right)+\frac{1}{4} \sum_{i=1}^{2} \lambda_{i} e^{\lambda_{i} \phi} F_{i}^{2}+2 \lambda_{0} \Lambda e^{\lambda_{0} \phi} \\
0=\nabla_{\mu}\left(e^{\lambda_{3} \phi} \nabla^{\mu} \chi_{1}\right), \quad 0=\nabla_{\mu}\left(e^{\lambda_{3} \phi} \nabla^{\mu} \chi_{2}\right) \\
0=\nabla_{\mu}\left(e^{\lambda_{1} \phi} F_{1}^{\mu \nu}\right), \quad 0=\nabla_{\mu}\left(e^{\lambda_{2} \phi} F_{2}^{\mu \nu}\right) .
\end{gathered}
$$

The metric ansatz is given by, $\quad d s^{2}=r^{\theta}\left(-r^{2 z} f(r) d t^{2}+\frac{d r^{2}}{r^{2} f(r)}+r^{2}\left(d x^{2}+d y^{2}\right)\right)$

where $\theta$ is the hyperscaling violation term and $z \neq 1$ is the Lifshitz scaling. The black hole factor is given by, $f(r)=1-\frac{m}{r^{\theta+z+2}}+\frac{Q_{2}^{2}}{2(\theta+2)(\theta+z) r^{2(\theta+z+1)}}+\frac{\alpha^{2}}{(\theta+2)(z-2) r^{\theta+2 z}}$ where ' $\mathrm{m}$ ' is mass, ' $Q_{2}$ ' is charge of black hole and ' $\alpha$ ' is the strength of momentum relaxation. The parameters of the gravity system are fixed considering the Null energy condition as,

$$
\begin{array}{rr}
\gamma=\sqrt{(\theta+2)(\theta+2 z-2)}, & \lambda_{0}=\frac{-\theta}{\gamma}, \quad \lambda_{1}=\frac{-(4+\theta)}{\gamma} \\
\lambda_{2}=\frac{(\theta+2 z-2)}{\gamma}, \quad \lambda_{3}=\frac{-\gamma}{\theta+2}, & Q_{1}=\sqrt{2(z-1)(\theta+z+2)}
\end{array}
$$


with $\Lambda=\frac{-1}{2}(\theta+z+1)(\theta+z+2)$ and $\phi=\gamma \log r$. The temporal component of both the gauge fields in the bulk are given by,

$$
\left(A_{1}\right)_{t}^{\prime}=Q_{1} r^{z-3-\lambda_{1} \gamma} \quad \text { and } \quad\left(A_{2}\right)_{t}^{\prime}=Q_{2} r^{z-3-\lambda_{2} \gamma} .
$$

Hawking temperature is given by, $T=\frac{f^{\prime}\left(r_{h}\right)}{4 \pi}=\frac{(2 z+1) r_{h}^{z}}{4 \pi}-\frac{Q_{2}^{2} r_{h}^{3}}{8 \pi(z+1)}-\frac{\alpha^{2} r_{h}^{2 z-1}}{4 \pi(z+1)}$. Applying holographic formalism we introduce the linear perturbations in the gauge fields, axion field and metric components as shown,

$$
\begin{array}{r}
\delta A_{i}(t, r)=\int_{-\infty}^{\infty} \frac{d \omega}{2 \pi} a_{i}(r) e^{-i \omega t}, \delta \chi_{i}(t, r)=\int_{-\infty}^{\infty} \frac{d \omega}{2 \pi} b_{i}(r) e^{-i \omega t} \\
\delta g_{t i}(t, r)=\int_{-\infty}^{\infty} \frac{d \omega}{2 \pi} r^{\theta+2} h_{t i}(r) e^{-i \omega t} .
\end{array}
$$

Considering perturbations in both the gauge fields we obtain second-order coupled differential equations. For simplification, we consider, $\Phi=\frac{r^{5-z} f b^{\prime}}{i \omega}$ and the equations of motion in the changed form is given by,

$$
\begin{aligned}
\left(r^{3-z-\theta} f a_{1}^{\prime}\right)^{\prime}+\frac{\omega^{2} a_{1}}{r^{z+5+\theta} f}+\frac{Q_{1}}{r^{z+5+\theta}}\left(Q_{1} a_{1}+Q_{2} a_{2}-a \Phi\right) & =0 \\
\left(r^{3 z-1+\theta} f a_{2}^{\prime}\right)^{\prime}+\frac{\omega^{2} a_{2}}{r^{3-z-\theta} f}+\frac{Q_{2}}{r^{5-z+\theta}}\left(Q_{1} a_{1}+Q_{2} a_{2}-a \Phi\right) & =0 \\
\left(r^{3 z-3} f \Phi^{\prime}\right)^{\prime}+\frac{\omega^{2}}{r^{5-z f}} \Phi-\frac{\alpha}{r^{5-z+\theta}}\left(Q_{1} a_{1}+Q_{2} a_{2}-a \Phi\right) & =0 .
\end{aligned}
$$

Multiplying (20) with $\frac{Q_{i}}{\alpha}$ and adding to (18) and (19) respectively we obtain the conserved current $J_{i}$ for the gauge fields,

$$
J_{1}=-r^{z-3-\theta} f a_{1}^{\prime}-\frac{Q_{1}}{\alpha} r^{3 z-3} f \Phi^{\prime}, \quad J_{2}=-r^{3 z-1+\theta} f a_{2}^{\prime}-\frac{Q_{2}}{\alpha} r^{3 z-3} f \Phi^{\prime} .
$$

Next, using the Onsager relation $(J=\tau X)$ we obtain the flow equations. Here, ' $\mathrm{J}$ ' are the response of the system from external sources ' $\mathrm{X}$ ' and $\tau$ contain transport coefficients. We evaluate $\tau$ matrix using the formalism given in [8]. We put the above coupled field equations in the given form,

$$
\tau=\left\|\begin{array}{c}
-r^{3-z-\theta} f a_{1}^{\prime} \\
-r^{3 z-1+\theta} f a_{2}^{\prime} \\
-r^{3 z-3} \phi^{\prime}
\end{array}\right\|\left\|\begin{array}{c}
i \omega a_{1} \\
i \omega a_{1} \\
i \omega \phi
\end{array}\right\|^{-1} .
$$

The radial derivative is given by,

$$
\tau^{\prime}=\left\|\begin{array}{c}
-r^{3-z-\theta} f a_{1}^{\prime} \\
-r^{3 z-1+\theta} f a_{2}^{\prime} \\
-r^{3 z-3} \phi^{\prime}
\end{array}\right\|\left\|\begin{array}{c}
i \omega a_{1} \\
i \omega a_{1} \\
i \omega \phi
\end{array}\right\|^{-1}-i \omega \tau\left\|\begin{array}{c}
a_{1}^{\prime} \\
a_{2}^{\prime} \\
\phi^{\prime}
\end{array}\right\|\left\|\begin{array}{c}
i \omega a_{1} \\
i \omega a_{1} \\
i \omega \phi
\end{array}\right\|^{-1} .
$$

In the near horizon limit, we obtain the flow equation from the above equation using $f\left(r_{h}\right)=0$,

$$
0=\left(\begin{array}{ccc}
\frac{-i \omega}{r_{h}^{z+5+\theta}} & 0 & 0 \\
0 & \frac{-i \omega}{r_{h}^{3-z-\theta}} & 0 \\
0 & 0 & \frac{-i \omega}{r_{h}^{5-z}}
\end{array}\right)+i \omega \tau_{h}\left(\begin{array}{ccc}
\frac{1}{r_{h}^{z-3-\theta}} & 0 & 0 \\
0 & \frac{1}{r_{h}^{3 z-1+\theta}} & 0 \\
0 & 0 & \frac{1}{r_{h}^{3 z-3}}
\end{array}\right) \tau_{h} .
$$


We evaluate $\tau_{h}$ matrix and used it to obtain first order flow equations from equation (22) in the near horizon limit,

$$
-r_{h}^{z+1} f a_{1}^{\prime}=i \omega a_{1}, \quad-r_{h}^{z+1} f a_{2}^{\prime}=i \omega a_{2}, \quad-r_{h}^{z+1} f \phi^{\prime}=i \omega \phi .
$$

To establish an explicit dependence of transport coefficients on the second gauge field we consider the first gauge field equation (18) in the near horizon limit for $\omega \rightarrow 0$ and obtain, $\Phi \alpha=q_{1} a_{1}+q_{2} a_{2}$. Substituting all the terms in the current expressions and using Ohm's law as, $\sigma_{i j}=\frac{\partial J_{i}}{\partial E_{j}}$ we evaluate the DC conductivity as,

$$
\sigma_{11}=\frac{1}{r_{h}^{4+\theta}}+\frac{q_{1}^{2} r_{h}^{2 z-4}}{\alpha^{2}}, \quad \sigma_{12}=\frac{q_{1} q_{2} r_{h}^{2 z-4}}{\alpha^{2}}=\sigma_{21}, \quad \sigma_{22}=r_{h}^{2 z-2+\theta}+\frac{q_{2}^{2} r_{h}^{2 z-4}}{\alpha^{2}} .
$$

Also, since $a_{1}$ is the auxiliary field in the system, we can set $J_{1}=0$ [7] in the near horizon limit and obtain, $\sigma_{D C}=r_{h}^{2 z-2+\theta}\left[1+\frac{q_{2}^{2}}{r_{h}^{2+\theta}\left(\alpha^{2}+q_{1}^{2} r_{h}^{2 z+\theta}\right)}\right]$. For $\alpha \rightarrow 0$ we obtain a constant conductivity from $\sigma_{D C}$. Also, we noticed the conductivity is a combination of two different components, a charge-conjugation symmetric term and the dissipation term [9]. The explicit dependence of conductivity on Lifshitz scaling(z) and $\theta$ is observed. Here, we summarize our main findings of this work as,

a) At high temperature $\left(q_{2}=0\right)$, we obtain linear resistivity $\left(\rho_{11}=\frac{1}{\sigma_{11}}\right)$ for $z=4+\theta$ and $-4<\theta<8 / 3$.

b) At low temperature $\left(q_{2} \neq 0\right)$, we obtain linear resistivity keeping $\theta=0$ and $z=4 / 3$.

c) For $J_{1}=0$, the temperature dependence of the DC conductivity is given by, $\sigma_{D C} \sim T^{\frac{2 z-2+\theta}{z}}+\frac{q_{2}^{2}}{q_{1}^{2}} T^{\frac{-\theta-4}{z}}+\alpha^{2} T^{\frac{-2}{z}}$

\section{Conclusions and Discussions}

In this report, we discussed the applications of holography to investigate strongly coupled systems which otherwise were inaccessible. We evaluated the frequency response of conductivity using soft-wall model for $(1+1)$-dimensional systems. We also obtained the expression of DC conductivities for $(2+1)$-dimensional non-relativistic condensed matter systems after introducing the perturbations in both the gauge fields. Temperature dependence of DC conductivity for these systems has been explored. Further, we have studied the effect of the external magnetic field on DC transport[10].

\section{References}

1. J. M. Maldacena, Adv. Theor. Math. Phys. 2, 231 (1998).

2. S. S. Gubser, Phys. Rev. D 78, 065034 (2008)

3. S. A. Hartnoll, et. al. Phys. Rev. Lett. 101, 031601 (2008).

4. D. Maity et. al., Nucl. Phys. B 839, 526 (2010).

5. J. Ren, JHEP 1011, 055 (2010).

6. S. S. Afonin and I. V. Pusenkov, Eur. Phys. J. C 76, no. 6, 342 (2016).

7. S. Cremonini, H. S. Liu, H. Lu and C. N. Pope, JHEP 1704, 009 (2017).

8. Y. Tian, et.al., Phys. Rev. D 96, no. 4, 046011 (2017).

9. M. Blake and A. Donos, Phys. Rev. Lett. 114, no. 2, 021601 (2015).

10. N. Bhatnagar and S. Siwach, arXiv:1707.04013 [hep-th]. 